\title{
A Complete Augmented Reality Guidance System for Liver Punctures: First Clinical Evaluation
}

\author{
S.A. Nicolau ${ }^{2}$, X. Pennec ${ }^{1}$, L. Soler ${ }^{2}$, and N. Ayache ${ }^{1}$ \\ 1 INRIA Sophia, Epidaure, 2004 Rte des Lucioles, F-06902 Sophia-Antipolis, Cedex \\ \{Stephane.Nicolau, Xavier.Pennec, Nicholas.Ayache\}@sophia.inria.fr \\ 2 IRCAD-Hopital Civil, Virtual-surg, 1 Place de l'Hopital, 67091, Strasbourg, Cedex \\ \{stephane.nicolau, luc.soler\}@ircad.u-strasbg.fr
}

\begin{abstract}
We provided in 14 an augmented reality guidance system for liver punctures, which has been validated on a static abdominal phantom [16. In this paper, we report the first in vivo experiments.

We developed a strictly passive protocol to directly evaluate our system on patients. We show that the system algorithms work efficiently and we highlight the clinical constraints that we had to overcome (small operative field, weight and sterility of the tracked marker attached to the needle...). Finally, we investigate to what extent breathing motion can be neglected for free breathing patient. Results show that the guiding accuracy, close to $1 \mathrm{~cm}$, is sufficient for large targets only (above $3 \mathrm{~cm}$ of diameter) when the breathing motion is neglected. In the near future, we aim at validating our system on smaller targets using a respiratory gating technique.
\end{abstract}

\section{Introduction}

The treatment of liver tumors by Radio-Frequency (RF) is a new technique which begins to be widely used [11. The guidance procedure to reach the tumors with the electrode is generally made visually with per-operative $2 \mathrm{D}$ cross-sections of the patient using either Ultra-Sound (US), Computed Tomography (CT) or Magnetic Resonance Images (MRI). Practitioners empirically evaluate their targeting accuracy around $3 \mathrm{~mm}$. However, the insertion needs repetitive CT/MRI images for needle adjustment (if CT/MRI guided) and sometimes several reinsertion attempts. This lengthens the intervention duration, and increases post procedure complications and radiation exposure (when CT-guided). Moreover, the targeting accuracy depends on the practitioner's experience.

Some systems have already been designed for puncture application. However they are quite expensive, or unsuitable for liver punctures. Some of them need a robotic arm [4,7] or a head mounted display associated to a cumbersome reference structure attached to the MRI table [18. Others 6] guide the needle placement when it is contained in a single axial CT slice. Their system is suited to the majority of cases but not to liver punctures. Indeed, most of liver punctures 
cannot be realized along an axial plane because of the critical structures that have to be avoided (like vessels). Finally, the C-arm systems presented in [2,12] do not allow to visualize what structures are on the needle path, which prevents their use for liver punctures.

To help practitioners, we developed in [14,16] a low cost augmented reality (AR) guidance system for CT/MRI assisted liver punctures: only one PC, two cameras and some radio-opaque markers are necessary. Despite breathing motion, we choose to use a rigid model since the patient is usually under general anesthesia and intubated to limit the pain (60\% of CT-guided RF ablation are done under general anesthesia at the Strasbourg Hospital). Thus it is possible to ask for a breath control that can provide a tumor repositioning error about $1 \mathrm{~mm}$ (see [20,1, 19]). This system was fully validated on a static abdominal phantom [14, 16]: several surgeons and engineers were able to reach targets with an accuracy of $2 \mathrm{~mm}$ and a duration under $40 \mathrm{sec}$.

In this paper, we report the first introduction of our system in the operating room (OR) during interventions on patients. After a presentation of our system, we explain how we evaluate its accuracy on patient with a safe protocol that we developed. Then, we present encouraging results which show that a full validation is possible with few additional constraints in the OR.

\section{System Components}

In our setup, two jointly calibrated cameras are viewing the skin of the patient who is lying on the CT-table. A 3D model of the patient (including his skin, liver and tumors) is automatically obtained from the first CT scan [17], and is rigidly registered in the camera frame thanks to radio-opaque markers previously stuck on the patient's skin. The needle being tracked by the cameras, we display on a screen its relative position with respect to the patient model.

In this section, we summarize the basic algorithmic principles of our system. Firstly, we explain how we automatically extract and match the radio-opaque markers (to avoid time loss). Then, we describe the $3 \mathrm{D} / 2 \mathrm{D}$ criterion we use to register the $3 \mathrm{D}$ patient model in the camera frame, and show how we track the needle position in real-time. Finally, we present the guiding interface designed with surgeons.

\subsection{Automated Data Processing}

The principle of the marker localization in the video images is based on a HSV color analysis, followed by a component size and shape thresholding. The markers in the CT-image are extracted by a top-hat characterization that emphasizes small singularities on the skin surface.

The matching between the video markers is realized with epipolar geometry, and the correspondences between video and CT markers are carried out by a prediction/verification algorithm. A validation study [14] showed that these algorithms are robust and that the overall computation time of the extraction and 
matching process is below $120 \mathrm{sec}$ (CT marker extraction - done once - takes 118 sec. on average, video marker tracking and registration are refreshed at $10 \mathrm{~Hz}$ ).

\subsection{Registration of the Virtual Model in the Camera's Frame}

We choose a $3 \mathrm{D} / 2 \mathrm{D}$ point registration approach to provide the rigid transformation that relates scanner frame and camera frame. The classical choice is to optimize the SPPC criterion (see [15]):

$$
\operatorname{SPPC}(T)=\sum_{k=1}^{S} \sum_{i=1}^{N} \xi_{i}^{k} \cdot \frac{\left\|\tilde{m}_{i}^{(k)}-P^{(k)}\left(T \star \tilde{M}_{i}\right)\right\|^{2}}{2 \cdot \sigma_{2 D}}
$$

where $S$ (resp. $N$ ) is the number of cameras (resp. markers), $\tilde{m}_{i}^{(k)}$ is the observed $2 \mathrm{D}$ coordinates of the $\mathrm{i}^{t h}$ markers in the $\mathrm{k}^{t h}$ video image, $\tilde{M}_{i}$ is the observed $3 \mathrm{D}$ coordinates of the $\mathrm{i}^{t h}$ markers in the CT-image, $P^{(k)}$ the projective function, $\xi_{i}^{k}$ is a binary variable equal to 1 if the $\mathrm{i}^{\text {th }}$ marker is visible in the $\mathrm{k}^{\text {th }}$ video image and 0 if not, and $T$ the sought transformation. However, this criterion considers that noise only corrupts the $2 \mathrm{D}$ data and that $3 \mathrm{D}$ data are exact. In our context, this assumption is erroneous as the marker extraction from the CT-image is corrupted by noise as well.

A more realistic statistical hypothesis is that we are measuring noisy versions $\tilde{M}_{i}$ of the unknown exact $3 \mathrm{D}$ points $M_{i}$ (more details are given in [15]). A ML estimation of the transformation $T$ and the auxiliary variables $M_{i}$ leads to minimize the Extended Projective Points Criterion (EPPC):

$$
\operatorname{EPPC}\left(T, M_{1}, \ldots M_{N}\right)=\sum_{i=1}^{N} \frac{\left\|\tilde{M}_{i}-M_{i}\right\|^{2}}{2 \cdot \sigma_{3 D}{ }^{2}}+\sum_{k=1}^{S} \sum_{i=1}^{N} \xi_{i}^{k} \cdot \frac{\left\|\tilde{m}_{i}^{(k)}-m_{i}^{(k)}\right\|^{2}}{2 \cdot \sigma_{2 D}{ }^{2}}
$$

\subsection{Needle Tracking}

To track the needle location and orientation in the camera frame, we attach an oriented square marker whose corners are automatically localized on video images in real-time using an adapted version of the ARTkit library [9. Then, knowing the size of the square, we are able to localize it in the camera reference frame by minimizing the classical 3D/2D SPPC criterion. Calibrating the relative needle position with respect to the square marker with the pivot method [10], we are finally able to superimpose the virtual model on the real one on video images. An accuracy evaluation realized in simulated clinical conditions (cameras $1.5 \mathrm{~m}$ away from the needle with a test volume of $40 \times 30 \times 25 \mathrm{~cm}^{3}$ ), showed that the average superimposition error of a radiofrequency needle was $0.95 \mathrm{~mm}$.

\subsection{A Secured and Ergonomic Guidance Interface}

Our interface has been optimized with surgeons, in order to provide them a clear and intuitive tool. It is divided into three screens (see Fig. 1) described below. 
The bottom left screen displays one of the two video images returned by our cameras, on which can be superimposed the virtual needle and the $3 \mathrm{D}$ patient model. The user can check visually the registration quality by superimposing virtual elements (like skin and radio-opaque markers). In the right screen, the $3 \mathrm{D}$ virtual scene, composed of the $3 \mathrm{D}$ patient model and the needle representation, is rendered from a viewpoint controlled by the

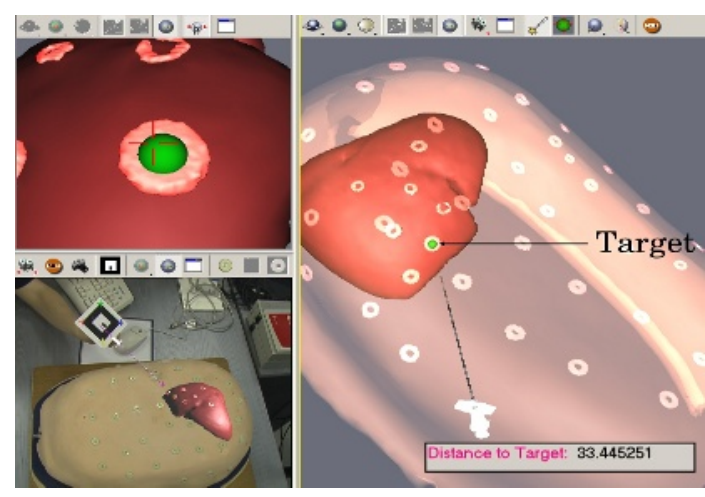

Fig. 1. Illustration of the three screen guidance interface user. Moreover, it is possible to display the CT-scan from which the reconstruction is made, and navigate through its slices. In the top left screen, we display a view that corresponds to what would see a camera positioned on the needle tip and oriented along its axis. This view facilitates the orientation of the needle toward the point to be reached before its insertion.

\section{Clinical Experiments on Patients}

We have previously evaluated the whole system on an abdominal phantom [16], and we have shown that, with simulated clinical conditions, our system accuracy and ergonomy are fully validated. However, in an OR, we will undergo additional constraints (lack of room, luminosity variation, validity range of rigidity assumption) that may hamper marker extraction, needle tracking and 3D model registration accuracy.

To check the efficiency of a system in real clinical conditions, the standard procedure is to make several tests on animals (like Vogt [18] or Mourgues [13]). However, it does take a long time to get animal study authorizations (at least 6 months in Strasbourg). Moreover, animals are not optimal models for evaluation: their anatomy size and shape, skin color, vessel branching structure and tissue mechanical properties are different from human ones. Thus, we propose to introduce our system in the OR directly on human patients, thanks to a strictly passive setup: the intervention is realized with the setup needed by our system, yet with the standard CT-guided protocol. In these conditions, no ground truth is available to evaluate the accuracy of our system (invasive technique are needed, which is not clinically acceptable). Thus, we designed a protocol that allows to assess the system accuracy w. r. t. the practitioner accuracy, without any risk for the patient.

In the sequel, we firstly describe the evaluation protocol we developed. Then we present the first experiments we realized on patients. Let us highlight that these experiments are not aimed at validating the system but just at evaluating 
it to determine the problems that need to be overcome to introduce it in the OR. The standard protocol was not modified, which means that patients breathed freely during the interventions. Then, it allowed us to see to what extent breathing motion could be neglected or not.

\subsection{A Safe Validation Protocol with Patients}

The purpose of the method is to measure the distance between the real tumor and the virtual tumor registered by our system. Since no ground truth is available (without modifying the intervention protocol with an invasive procedure) for the real tumor position, we consider the final position of the needle as a reference. Then, we measure, at the end of the needle insertion, the distance between the needle tip and the target registered by our system. This target is a point $C_{\text {scan }}$ within the tumor that the practitioner defines (in the first acquisition $C T_{0}$ ) as the point he is targeting. Obviously, our reference depends on the practitioner's accuracy. Radiologists and surgeons evaluate their targeting acurracy to $3 \mathrm{~mm}$. Therefore, to show the equivalence between our system and the practitioner, we have to obtain an average distance close to $3 \mathrm{~mm}$ (assuming that both practitioner targeting and system registration errors are unbiased).

Figure 2 summarizes the different steps of our protocol and how we get a measure. To be in our system setup, a square marker is attached to the needle, radio-opaque markers are stuck on the patient skin, and we set our two cameras in the OR. The practitioner performs the intervention with his radiological protocol (repetitive CT acquisitions) without any advice nor instruction from our system. Note that the radiation exposure is not negligible since 40 sets of 4 slices are at least taken for each tumor. When the needle insertion is completed, the practitioner checks visually (using a control scan) that the needle tip was sufficiently close to the initial target. This is done to avoid a bias. Then, we make a stereoscopic video acquisition of the patient abdomen (with the radio-opaque

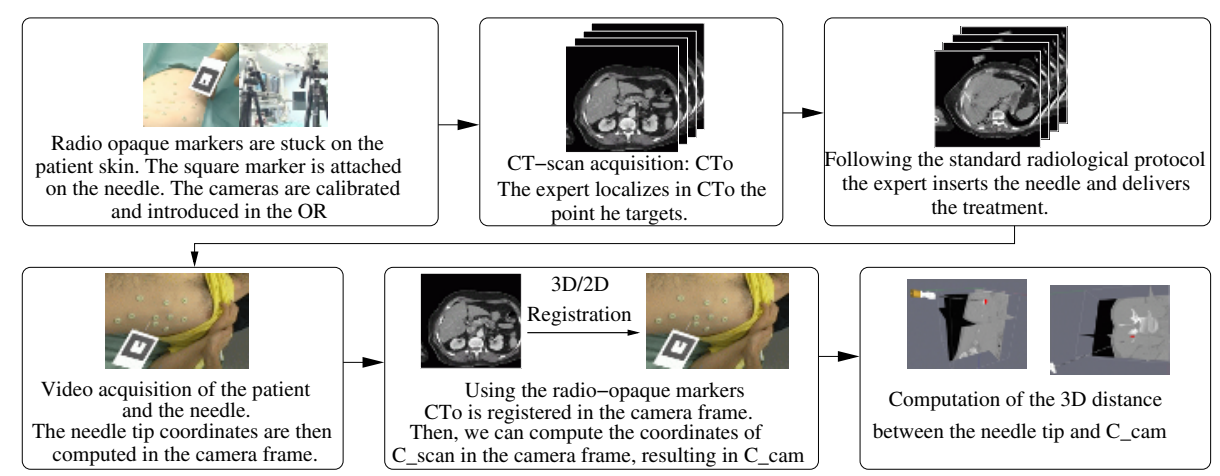

Fig. 2. Description of the validation protocol without any risk for the patient. It allows to obtain an assessment of the system guidance error with respect to the targeting error of the practitioner. 
markers) and of the square marker attached to the needle. From this acquisition, we estimate the needle tip position $P_{c a m}$ and register the target $C_{\text {scan }}$ (originally defined in $C T_{0}$ ) in the camera frame. The registered target $C_{c a m}$ is the point toward which we would have guided the practitioner if he had used our system. Finally, our accuracy measure is given by the distance $d\left(P_{c a m}, C_{c a m}\right)$.

\subsection{Results}

We have introduced our system three times in the OR. All the data processing algorithms worked efficiently (marker extraction and matching, needle tracking, 3D/2D registration). Figure 3 shows several augmented images obtained during the interventions. Quantitative accuracy results are shown in table 1.

The measured average accuracy is $9.5 \mathrm{~mm}$, which is two to three times larger than the value needed to show the equivalence between our system and a practitioner. We think that most of the error can be attributed to the free breathing of the patient during the intervention. Studies showed that the liver moves over $1 \mathrm{~cm}$ with respect to the skin with free breathing [5]. This means that the 3D
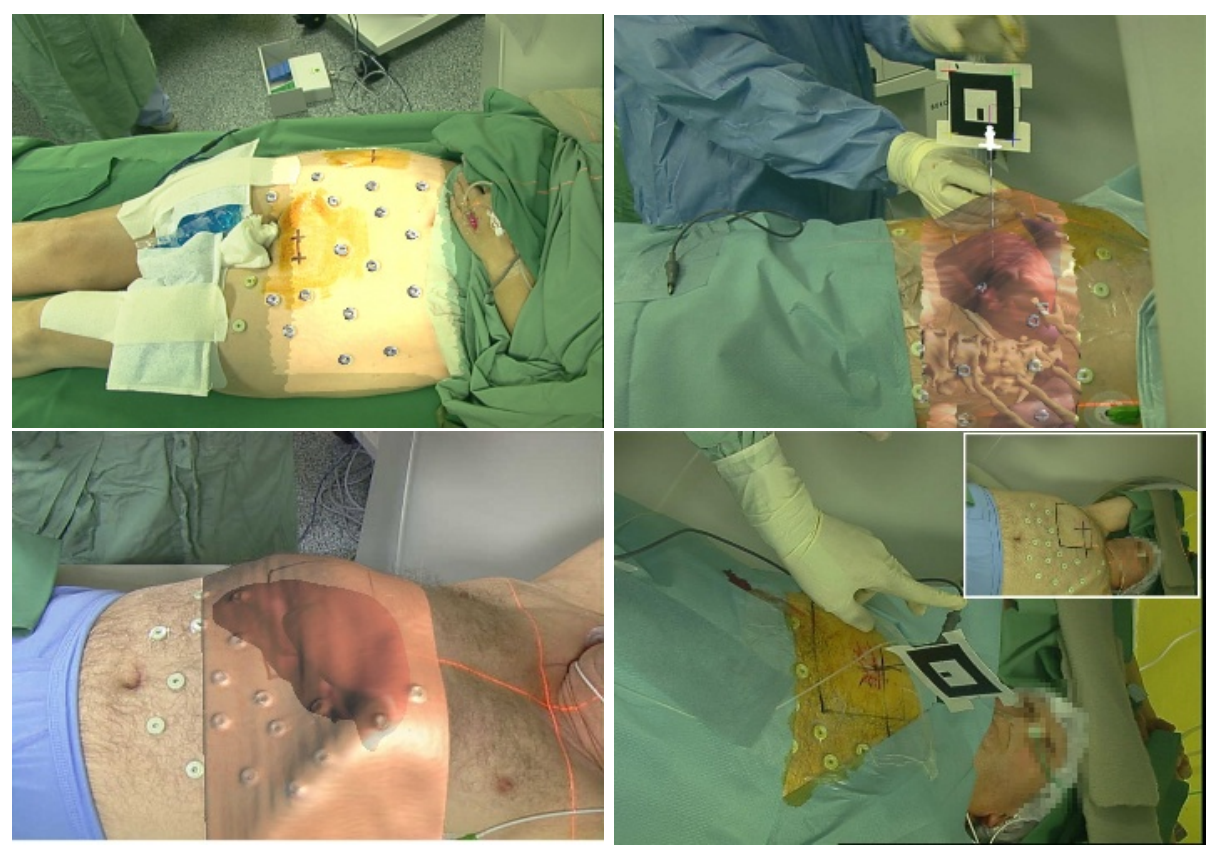

Fig. 3. Top left: visual check of the $3 \mathrm{D}$ model registration quality. The radio-opaque markers of the 3D reconstructed model are well superimposed on these visible in the video images. Top right: augmented view of the patient at the end of the needle insertion. Bottom left: view in transparency of the patient. The edge of the patient skin seemed perfectly superimposed with the $3 \mathrm{D}$ reconstruction edge. Bottom right: for this patient, only 7 markers were detectable among the 18 initially stuck. 
Table 1. Results of the system evaluation on three patients

\begin{tabular}{|c|c|c|c|c|c|}
\hline & $\begin{array}{c}\text { Tumor } \\
\text { type }\end{array}$ & $\begin{array}{c}\text { General } \\
\text { anesthesia }\end{array}$ & $\begin{array}{c}\text { Gas volume } \\
\text { monitoring }\end{array}$ & $\begin{array}{c}\text { Number of } \\
\text { markers used/stuck }\end{array}$ & $\begin{array}{c}\text { Accuracy } \\
\text { R-S (mm) }\end{array}$ \\
\hline \hline Patient 1 & Liver & no & no & $7 / 18$ & 12.0 \\
\hline Patient 2 & Bone & yes & no & $16 / 19$ & 6.7 \\
\hline Patient 3 : tumor 1 & Liver & no & no & $9 / 15$ & 8.0 \\
Patient 3 : tumor 2 & Liver & no & no & $9 / 15$ & 11.3 \\
\hline \hline Average & - & - & - & $10.6 / 17.3$ & 9.5 \\
\hline
\end{tabular}

model reconstructed from the initial CT-scan was not close enough to reality to obtain an accurate rigid registration.

The second error cause is the low number or radio-opaque markers used for the transformation computation. Indeed, although we stuck around 17 markers on the patient skin, only 10 on average were imaged in the initial CT-scan $C T_{0}$ (one can see on Fig. 3 that the 3D model does not contain all the radio-opaque markers). Moreover, on the first patient, the operative field was not large enough (for sterility reasons) and reduced the number of visible skin markers. This issue was solved afterward with the use of sterile transparent stickers that were stuck on the patient skin (see top right Fig. [3). Another explanation of the error is the relative position of the target with respect to the radio-opaque marker used for the registration. Since many unused markers were the closest ones to the target, the theoretical inaccuracy increased.

Note that we obtained our best accuracy result $(6.7 \mathrm{~mm})$ for the bone radiofrequency intervention. For this patient, the target was far away from the upper abdomen in a more static zone (see top left Fig. 31). Therefore, we are convinced that we can provide better results if a respiratory gating technique is used.

\section{Conclusion}

In previous papers [14,16], we developed and validated on phantom an AR system to guide liver percutaneous punctures in interventional radiology. This video based system tracks in real time the puncture needle and automatically registers (at a $10 \mathrm{~Hz}$ rate) the 3D patient model in the camera frame using radio-opaque markers. To show the robustness of the system in the OR (needle tracking, marker detection), we proposed to introduce it passively during interventions on patients. Since a passive approach limits the possibility of accuracy evaluation, we designed a new protocol that allows to assess the system accuracy without any risk for the patient. To our knowledge, it is the first time that an evaluation of AR-guided liver punctures is experimented on patients.

The three experiments we realized were an essential step to refine the constraints for using our system in a clinical setup. Firstly, we needed to build a square marker in sterile plastic for the needle tracking. On the one hand, it had to be sufficiently light to avoid curving the needle once inserted. On the other hand, its size had to be small to avoid touching the CT-gantry during the control 
scans (currently it is too large for an MRI gantry). Secondly, we have experimented problems with the positioning and visibility of the radio-opaque markers. They have to be stuck carefully in the liver range to be imaged in the first CTscan. Moreover, they are not visible in the video images if the practitioner does not enlarge the operative field (this could be realized with transparent sterile stickers). Finally, markers being non sterile, we could not stick them close to the entry point, inducing a loss of registration accuracy. This has to be compensated with a greater number of markers.

From a technical point of view, we showed that our system worked well in clinical conditions. However, we observed a positioning error which is larger than the practitioner's one, due to the breathing motion. We can take this motion into account either by modifying the clinical setup, i.e. by requiring a respiratory gating technique, or by modifying our system, for instance using a non-rigid model [3, 8]. Although the current accuracy of the final positioning is sufficient only for large targets (diameter above $3 \mathrm{~cm}$ ), practitioners estimate that the system provides relevant informations for orienting the needle at the beginning of the insertion. This initial guidance could avoid several insertion attempts.

In the next step, we will perform an evaluation with intubated patients under breathing control. Practitioner movements will be synchronized on the respiratory cycle point corresponding to the first CT acquisition. These experiments should show that the system can be used in pseudo static conditions.

We are currently adapting this work for laparoscopic application. We track the endoscopic camera and surgical tools to provide surgeons with AR view in the endoscope coupled with the relative position of tools. We hope to test that new system on static organs linked to the spine in less than six months. Eventually, since it would be more comfortable for patients and less complicated for practitioners to avoid respiratory gating techniques, we intend to use a nonrigid registration coupled with real time US images.

\section{References}

1. J.M. Balter et al, K.L. Lam, C.J. McGinn, T.S. Lawrence, and R.K. Ten Haken. Improvement of CT-based treatment-planning models of abdominals targets using static exhale imaging. Int. J. Radiation Oncology Biol. Phys., 41(4):939-943, 1998.

2. B. Bascle, N. Navab, M. Loser, B. Geiger, and R Taylor. Needle placement under x-ray fluoroscopy using perspective invariants. In $M M B I A^{\prime} 00$, pages 46-53, 2000.

3. J.M. Blackall et al. Tracking alignment of sparse ultrasound with preoperative images of the liver and an interventional plan using models of respiratory motion and deformation. In Proceedings SPIE Medical Imaging, 2004.

4. EM Boctor et al. Robotically assisted intraoperative ultrasound with application to ablative therapy of liver cancer. SPIE Med. Imaging 03, vol. 5029, pp. 281-291.

5. M. Clifford et al. Assessment of hepatic motion secondary to respiration for computer assisted interventions. Computer Aided Surgery, 7:291-299, 2002.

6. G. Fichtinger et al. Needle insertion in CT scanner with image overlay - cadaver studies. In MICCAI'04, LNCS 3217, pages 795-803. Springer-Verlag, 2004.

7. G. Fichtinger et al. System for robotically assisted prostate biopsy and therapy with intraoperative CT guidance. Journal of Academic Radiology, 9(1):60-74, 2002. 
8. D.J. Hawkes et al. Tissue deformation and shape models in image-guided interventions: a discussion paper. Medical Image Analysis, 9(2):163-175, 2004.

9. Hiro. Human interface technology lab., http://www.hitl.washington.edu/artoolkit/.

10. S. Lavalle, P. Cinquin, and J. Troccaz. Computer Integrated Surgery and Therapy: State of the Art, chapter 10, pages 239-310. IS Press, Amsterdam, NL, in C. Roux and J.L. Coatrieux edition, 1997.

11. J.F. McGahan and G.D. Dodd III. Radiofrequency ablation of the liver: Current status. American Journal of Roentgenology, 176(1):3-16, 2001.

12. M Mitschke, A. Bani-Hashemi, and N. Navab. Interventions under videoaugmented x-ray guidance: Application to needle placement. In MICCAI'00, pages 858-868, Pittsburgh, Pennsylvania USA, October 11-14 2000.

13. Fabien Mourgues, Thierry Vieville, Volkmar Falk, and Ève Coste-Manire. Interactive guidance by image overlay in robot assisted coronary artery bypass. In MICCAI'03, LNCS 2878, pp. 173-181, November 2003.

14. S. Nicolau, A. Garcia, X. Pennec, L. Soler, and N. Ayache. An augmented reality system to guide radio-frequency tumor ablation. In the Journal of Computer Animation and Virtual World, 16(1):1-10, 2005.

15. S. Nicolau, X. Pennec, L. Soler, and N. Ayache. An accuracy certified augmented reality system for therapy guidance. In ECCV'04, LNCS 3023, pages 79-91. 2004.

16. S. Nicolau, J. Schmid, X. Pennec, L. Soler, and N. Ayache. An augmented reality $\&$ virtuality interface for a puncture guidance system: Design and validation on an abdominal phantom. In MIAR 2004, LNCS 3150, pp. 302-310, 2004.

17. L. Soler, H. Delingette, G. Malandain, N. Ayache, and J. Marescaux. Fully automatic anatomical, pathological, and functional segmentation from CT scans for hepatic surgery. Computer Aided Surgery, 6(3), August 2001.

18. S. Vogt et al. Augmented reality system for MRI-guided interventions: phantom studies and first animal test. In SPIE Med. Imaging, vol. 5367, pp 100-109, 2004.

19. R. Wagman, E. Yorke, E. Ford, P. Giraud, G. Mageras, B. Minsky, and K.. Rosenzweig. Respiratory gating for liver tumors: use in dose escalation. Int. J. Radiation Oncology Biol. Phys., 55(3):659-668, 2003.

20. J. Wong, M. Sharpe, D. Jaffray, V. Kini, J. Robertson, J. Stromberg, and A. Martinez. The use of active breathing control (abc) to reduce margin for breathing motion. Int. J. Radiation Oncology Biol. Phys., 44(4):911-919, 1999. 